\title{
NUMBERS OF REPRESENTATIONS IN CERTAIN SENARY QUADRATIC FORMS*
}

\author{
BY E. T. BELL
}

1. Simplicity of Number of Representations. We first recall a definition from previous papers. $\dagger$ Let $N[n=f]$ denote the number of matrices $(x, y, z, \cdots)$ of integers $x, y, z, \cdots$, each $\gtreqless 0$, such that $n=f(x, y, z, \cdots)$; namely, $N[n=f]$ is the number of representations of $n$ in the form $f$. If $N[n=f]$ is a polynomial in the real divisors of $n$ alone, $N[n=f]$ is called simple, otherwise, compound. It is an interesting problem to determine those quadratic forms $f$ in $r$ variables for which $N[n=f]$ is simple, also the lightest restrictions that must be imposed upon $n$ in order that a compound $N[n=f]$ shall be simple for the restricted values of $n$. For example, if $f$ is a sum of 10 squares, $N[n=f]$ is compound if $n$ is an arbitrary positive integer, but $N[4 n+3=f]$ is simple, a theorem due to Eisenstein. By the means illustrated in the present paper, I have found numerous particular solutions of the above problem, also several general theorems (notably relating to sums of squares).

Write for a moment

$$
x^{2}+a y^{2}+b z^{2}+c w^{2}+d u^{2}+e t^{2}=(1, a, b, c, d, e) .
$$

It has long been known that the number of representations of $n$ in $(1,1,1,1,1,1)$ is simple, where $n$ is any integer $>0$. We shall find that the like holds for each of the 5 forms $(1,1,1,1,1,4)$, $(1,1,1,1,4,4),(1,1,1,4,4,4),(1,1,4,4,4,4),(1,4,4,4,4,4)$, with the single exception of the case $n=m \equiv 1 \bmod 4$, in which the number of representations in each of the 5 forms can easily be reduced to a polynomial in the real divisors of $m$ plus a constant multiple of the number of representations of $m(\equiv 1 \bmod 4)$ in

$$
x^{2}+y^{2}+z^{2}+w^{2}+u^{2}+16 t^{2} \text {. }
$$

\footnotetext{
* Presented to the Society, April 5, 1930.

† See in particular, a paper on quinary forms in the 1930 volume of the American Journal of Mathematics. The results quoted for 5 squares will be found in the same journal, vol. 42 (1920), pp. 185-186. These references cover $\$ 2$.
} 
I have not succeeded in proving or disproving that the last number is simple. The summary is in $\S 3$.

Before proceeding to the discussion, two remarks may be made. First, it is clearly unnecessary, as done here, to refer to results which were first deduced from identities between elliptic theta constants; all can be obtained by unimodular substitutions and correspondence from the known theorems for 6 squares which, as first shown by Liouville, are easily derivable from elementary considerations independent of elliptic functions. But the use of transcendental identities is suggestive and perhaps easier. Second, considerations precisely similar to those of this paper can be applied to the forms

$$
\left(1,2^{a}, 2^{b}, 2^{c}, 2^{d}, 2^{e}\right),
$$

where each of $a, b, \cdots, e$ is one of $0,1,2,3$; also, it can be used to obtain at least partial enumerations for such forms when one or more of $a, b, \cdots, e$ exceeds 3 . If in $(1, a, b, c, d, e)$, one or more of $a, b, \cdots, e$ is divisible by a prime $>2$, identities between theta functions (not merely constants) must be used, if the transcendental method is employed. This, however, does not concern us here.

2. Five Senary Quadratics. Let $n$ denote an arbitrary integer $>0$, and $m$ an odd integer $>0$. Write $N_{r}(n)=$ the number of representations of $n$ as a sum of $r$ squares; $\xi_{r}(n)=$ the excess of the sum of the $r$ th powers of all those divisors of $n$ that are $\equiv 1$ mod 4 over the like sum in which the divisors are $\equiv 3 \bmod 4$; $\xi_{r}{ }^{\prime}(n)=$ the excess of the sum of all those divisors of $n$ whose conjugate divisors are $\equiv 1 \bmod 4$ over the like sum in which the conjugates are $\equiv 3 \bmod 4$. Each of $x, y, z, w, u, t$ is a variable integer ranging from $-\infty$ to $\infty$. It is clear that $x, y, \cdots, t$ are blanks or "dummies" in $N[n=f(x, y, \cdots, t)]$. The forms $f_{i}$ are defined by

$$
\begin{aligned}
& f_{0}=x^{2}+y^{2}+z^{2}+w^{2}+u^{2}, \\
& f_{1}=x^{2}+y^{2}+z^{2}+w^{2}+4 u^{2}, \\
& f_{2}=x^{2}+y^{2}+z^{2}+4 w^{2}+4 u^{2}, \\
& f_{3}=x^{2}+y^{2}+4 z^{2}+4 w^{2}+4 u^{2}, \\
& f_{4}=x^{2}+4 y^{2}+4 z^{2}+4 w^{2}+4 u^{2} .
\end{aligned}
$$


Hence, obviously, for $j=0,1, \cdots, 4$,

(1) $N\left[n=f_{4-j}+t^{2}\right]=N\left[n=f_{5-j}+t^{2}\right]$

$$
+N\left[n=f_{4-j}+t^{2} ; t \text { odd }\right] \text {, }
$$

since in the representations enumerated on the left $t$ may be even $\left(=2 t^{\prime}\right)$ or odd, and $N\left[n=f_{4-j}+4 t^{2}\right]=N\left[n=f_{5-j}+t^{2}\right]$. If in (1), the second $N$ on the right is known, and the value of the first $N$ on the right is given for $j=0$, then (1) is a completely solvable difference equation for $N\left[n=f_{4-j}+t^{2}\right]$. This is the case for the forms with which we shall be concerned.

Denote by $\phi(n)$ the number of representations of $n$ as a sum of 5 squares, $N_{5}(n)=\phi(n)$.

From the second reference in $\$ 1$, we have

$$
m \equiv 3 \bmod 4: \quad \sum \phi\left(m-4 a^{2}\right)=-10 \xi_{2}(m),
$$

the summation referring to $a=0, \pm 1, \pm 2, \cdots$, and continuing so long as $4 a^{2}<m$;

$$
\sum \phi\left(2 m-4 a^{2}\right)=40 \xi_{2}^{\prime}(m),
$$

where, by the notation, $m$ is an arbitrary odd integer $>0$, and $\Sigma$ is as in (2), $4 a^{2}<2 m$;

(4) $\quad m \equiv 3 \bmod 4: \quad \sum \phi(m-4 b(b+1))=80 \xi_{2}^{\prime}\left(\frac{m+1}{4}\right)$,

where $\Sigma$ refers to $b=0,1,2, \cdots$, and continues so long as $4 b(b+1)<m$.

From the second reference, we shall require, with $j=0,1,2,3,4$,

$$
\begin{gathered}
m \equiv 3 \bmod 4: \quad N\left[m=f_{4-j}\right]=\frac{1}{60} j(j-1)(j+1) \phi(m) ; \\
N\left[2 m=f_{4-j}\right]=\frac{1}{20} j(j+1) \phi(2 m) .
\end{gathered}
$$

Combining (2) and (5), (3) and (6), (4) and (5), and applying (1) to the last pair, we easily find the results summarized in §3. For the last, we require also

$$
N_{6}(n)=4\left[4 \xi_{2}^{\prime}(n)-\xi_{2}(n)\right],
$$

cited from the second reference. 
3. Summary for the Forms of $\$ 2$. We find, for $j=0,1,2,3,4$, the following:

$$
\begin{aligned}
& m \equiv 3 \bmod 4: N\left[m=f_{5-j}+t^{2}\right]=-\frac{1}{6} j(j-1)(j+1) \xi_{2}(m) ; \\
& m \equiv 1 \bmod 2: N\left[2 m=f_{5-j}+t^{2}\right]=2 j(j+1) \xi_{2}^{\prime}(m) \\
& n \text { arbitrary: } N\left[4 n=f_{4-j}+t^{2}\right] \\
& =\frac{2}{3}\left(j^{4}+2 j^{3}-j^{2}-2 j+24\right) \xi_{2}^{\prime}(n)-4 \xi_{2}(n) .
\end{aligned}
$$

The second is valid also for $j=5$ if $m \equiv 1 \bmod 4$; the first holds also when $j=5$. It will be sufficient to prove the last, as the proofs of the others are similar and simpler.

4. Proofs. From $\$ 3(4)$, we have at once, on applying (5),

$$
\sum N\left[m-4 b(b+1)=f_{4-j}\right]=\frac{4}{3} j(j-1)(j+1) \xi_{2}^{\prime}\left(\frac{m+1}{4}\right) .
$$

But here $m=4 h+3, h \geqq 0$. Hence $m+1=4(h+1)=4 n$, and the above is equivalent to

$$
N\left[4 n=f_{4-j}+t^{2} ; t \text { odd }\right]=\frac{8}{3} j(j-1)(j+1) \xi_{2}^{\prime}(n) ;
$$

and hence, by (1),

$$
N\left[4 n=f_{4-j}+t^{2}\right]=N\left[4 n=f_{5-j}+t^{2}\right]+\frac{8}{3} j(j-1)(j+1) \xi_{2}^{\prime}(n) .
$$

But we have evidently

$$
N\left[4 n=f_{5}+t^{2}\right]=N\left[4 n=f_{5}+4 t^{2}\right]=N_{6}(n),
$$

since in $4 n=f_{5}+t^{2}, t$ is necessarily even. Thus

$$
N\left[4 n=f_{5}+t^{2}\right]=4\left[4 \xi_{2}^{\prime}(n)-\xi_{2}(n)\right] .
$$

Taking $j=0,1,2,3,4$ in the above, we find five results which are easily stated as the single formula given.

California Institute of Technology 